\title{
Interrogation of fibre Bragg gratings through a fibre optic rotary joint on a geotechnical centrifuge
}

\author{
Ricardo Correia $^{\mathrm{a}}$, Stephen W. James ${ }^{\mathrm{b}}$, Alec Marshall ${ }^{\mathrm{c}}$, Charles Heron ${ }^{\mathrm{c}}$, Sergiy Korposh* ${ }^{\mathrm{a}}$ \\ ${ }^{a}$ Advanced Optics group, Electrical Systems and Optics Research Division, University of \\ Nottingham, Nottinghamshire, NG7 2RD, UK; ${ }^{b}$ Dept. of Engineering Photonics, School of \\ Engineering, Cranfield University, Bedfordshire, MK43 0AL, UK.; ' Department of Civil \\ Engineering, University of Nottingham, University Park, Nottingham NG7 2RD, UK
}

\begin{abstract}
The monitoring of an array of fibre Bragg gratings (FBGs) strain sensors was performed through a single channel, single mode fibre optic rotary joint (FORJ) mounted on a geotechnical centrifuge. The array of three FBGs was attached to an aluminum plate that was anchored at the ends and placed on the model platform of the centrifuge. Acceleration forces of up to $50 \mathrm{~g}$ were applied and the reflection signal of the monitored FBGs recorded dynamically using a $2.5 \mathrm{kHz}$ FBG interrogator placed outside the centrifuge. The use of a FORJ allowed the monitoring of the FBGs without submitting the FBG interrogator to the high g-forces experienced in the centrifuge.
\end{abstract}

Keywords: FBGs, geotechnical centrifuge, fibre optic rotary joint.

\section{INTRODUCTION}

In geotechnical engineering, the use of small scale models tested on a geotechnical centrifuge represents a relatively inexpensive way of studying the behavior of soils and soil-structure interactions. The scaled models are subjected to high accelerations when placed at the end of a centrifuge arm. This results in the increase of the self-weight of the materials within the model by a factor $\mathrm{N}$ compared to $1 \mathrm{~g}$, where $\mathrm{g}$ is gravity and $\mathrm{N}$ is controlled by the rotational speed of the centrifuge. The increase of self-weight allows replication of full-scale stresses within the model soil, which is required in order to accurately replicate full-scale soil behavior (i.e. strength, stiffness) within the model. Geotechnical centrifuge testing has proven invaluable for the study of many problems in Civil Engineering, including foundations for bridges and buildings, stability of slopes and seawalls, and deformations caused by excavations and tunneling. Geotechnical centrifuge testing also provides the ability to study the effects of earthquakes on buildings, bridges, roads and the ground upon which they stand.

The acquisition of high-quality measurement of engineering parameters (i.e. displacement, force, strain, pressure) is imperative for geotechnical centrifuge tests. It is important that instruments incorporated into the model are as small and light-weight as possible, since their weight also increases in the centrifuge, which may adversely affect the soil model. FBG technology represents an attractive alternative to conventional instrumentation methods used for geotechnical testing because of the small/lightweight nature of the fibres and the potential to acquire a large number of high-quality measurements (including strain and pressure).

This paper presents results obtained from an experiment in which FBGs were attached to an aluminum plate that was subjected to bending due to an increased acceleration field within a geotechnical centrifuge. The FBGs were interrogated from outside the centrifuge using a fibre optic rotary joint (FORJ). FORJs have been previously used to allow the monitoring of FBGs in different applications, such as composite propeller blade ${ }^{1}$ strain monitoring and for structural integrity health monitoring of wind turbine blades ${ }^{2}$. To our knowledge, this is the first time that FORJs have been used to monitor FBGs on a centrifuge, allowing the placement of the interrogator outside the centrifuge to avoid being subjected to high g-forces.

*s.korposh@ nottingham.ac.uk; www.nottingham.ac.uk

Sixth European Workshop on Optical Fibre Sensors, edited by Elfed Lewis, Proc. of SPIE Vol. 9916, 99162B - (C) 2016 SPIE · CCC code: 0277-786X/16/\$18 - doi: 10.1117/12.2236979 


\section{EXPERIMENTAL SETUP}

\subsection{Centrifuge}

The Nottingham Centre for Geomechanics (NCG) centrifuge is a typical medium-size beam centrifuge with one swinging platform at a radius of $2.0 \mathrm{~m}$. The capacity of the centrifuge is $50 \mathrm{~g}$-Ton; it is capable of accelerating a payload of $500 \mathrm{~kg}$ to an acceleration of $100 \mathrm{~g}$, and lesser payloads up to $150 \mathrm{~g}$. The major components of the machine are shown in Figure 1.

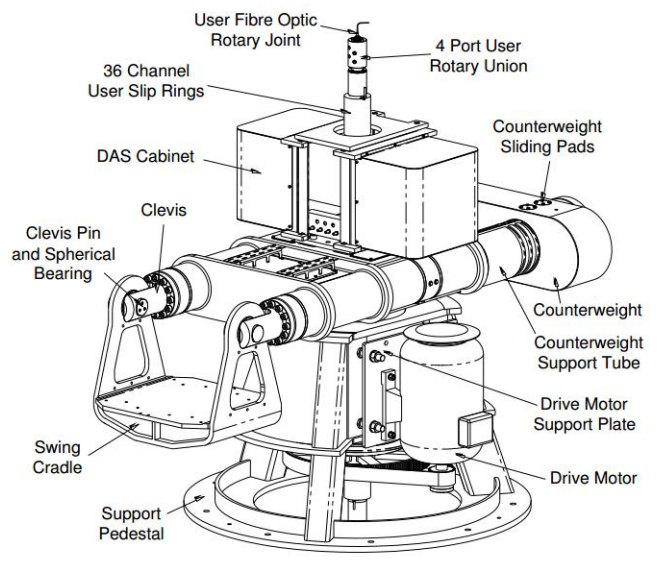

Figure 1: Illustration of the NCG geotechnical centrifuge ${ }^{3}$.

The machine consists of a fixed pedestal and rotating arms, a swing platform to house a model container, an electric motor for the control of rotational speed, cabinets mounted near the machine axis for housing data acquisition and electronic components, and a fibre optic rotary joint and slip rings for transmission of data and electrical signals. The inertial acceleration of a model is achieved through direct control of the rotational speed through a $75 \mathrm{~kW} 3$-phase motor, driven by a variable frequency inverter.

Balancing of the machine (i.e. achieving equivalent forces acting on either ends of the arms) is important to ensure smooth and safe operation. This is achieved by first using a fixed-mass counterweight prior to the centrifuge flight, then in-flight the machine automatically performs a fine-tuned balancing, to within $+/-50 \mathrm{kgm}$, by moving oil in the tubular rotor arms from one side of the main axis to the other. Out-of-balance forces are determined by a strain sensor sensitive to deflection of the support pedestal at the location of one of the legs.

\subsection{FBG sensors and interrogator}

Three FBG sensors were used in this experiment to measure strain of the aluminum plate. The sensors were fabricated using single mode optical fibre (Corning SM-28) that was hydrogen loaded at 130bar at room temperature for 2 weeks. A frequency quadrupled Nd:YAG laser emitting at $266 \mathrm{~nm}$ (Continuum Minilite I) was used to inscribe the gratings using the phase mask technique. The fabricated FBGs had a central wavelength of (1535nm, 1545nm and $1556 \mathrm{~nm})$, a reflectivity of $50 \%$ and a total length of $3 \mathrm{~mm}$. The FBGs were attached to a $340 \times 75 \mathrm{~mm}$ long aluminum plate using cyanoacrylate glue at positions $122 \mathrm{~mm}, 137 \mathrm{~mm}$ and $152 \mathrm{~mm}$ from the left of the plate. The plate was then positioned at the tip of centrifuge and anchored at its extremes with the side containing the sensors facing away from the centrifuge centre, such that FBGs would experience tensile loading. The fibre optic cable was then routed to the FORJ (Princitel, RST-155-28-SC) and secured carefully. The FBG interrogator used in this experiment was a commercial unit from Smartfibres (Smartscan). The unit is a 4 channel, high speed interrogator capable of scanning the entire wavelength range of $40 \mathrm{~nm}(1528-1568 \mathrm{~nm})$ at a scan frequency of $2.5 \mathrm{kHz}$. 


\section{RESULTS AND DISCUSSION}

Prior to applying increased gravitational forces to the test plate, the centrifuge was manually turned and the FBGs spectrum monitored. It was noted that as the centrifuge rotated, the intensity of the reflected signal of the FBG changed following a sinusoidal pattern, due to a slight eccentricity in the FORJ. A similar modulation was also observed in the central wavelengths of the returned by the interrogator software, which is believed to be a result of a limitation of the centroid algorithm used to identify the central wavelength of the Bragg peaks. The change in wavelength against time measured as the centrifuge arm was turned by two revolutions is shown in Figure 2a. Figure 2(b) shows the strain measured by the 3 FBGs as the centrifuge speed was varied from 0 to $150 \mathrm{rpm}$, equivalent to $150 \mathrm{~g}$ at the $2.0 \mathrm{~m}$ platform radius. The data shows that the strain measured as the centrifuge decelerated differed from that measured during the acceleration phase, which is thought to be a result of a relaxation of the anchoring points of the test plate. The sinusoidal modulation of the Bragg wavelength was present throughout the test, and its amplitude was $10 \mathrm{pm}$, irrespective of the centrifuge acceleration. An FFT analysis of the tracked FBG peaks as the centrifuge speed was increased is shown in Figure 3, showing clearly the relationship between the rotation rate and the frequency of the sinusoidal modulation on the Bragg wavelengths. We have observed similar issues when connecting the interrogation unit to FBGs using patch cords that are not mode matched to the fibre in which the FBGs are written (e.g. smf28 patch cord connecting to FBGs written in $\mathrm{B} / \mathrm{Ge}$ co-doped optical fibre). Movement of the patch cord was observed to cause modulation of the intensity of the peaks and produce an erroneous modulation of the Bragg wavelength determined by the algorithm.

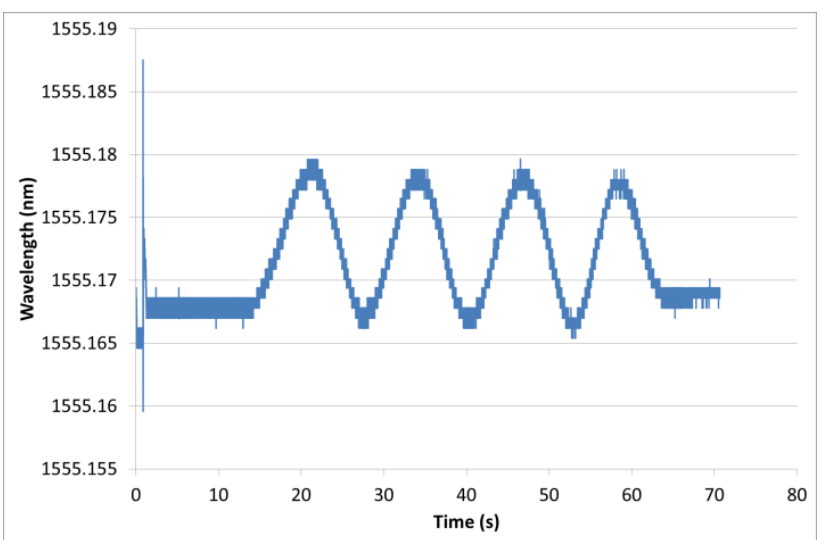

$\mathrm{a}$

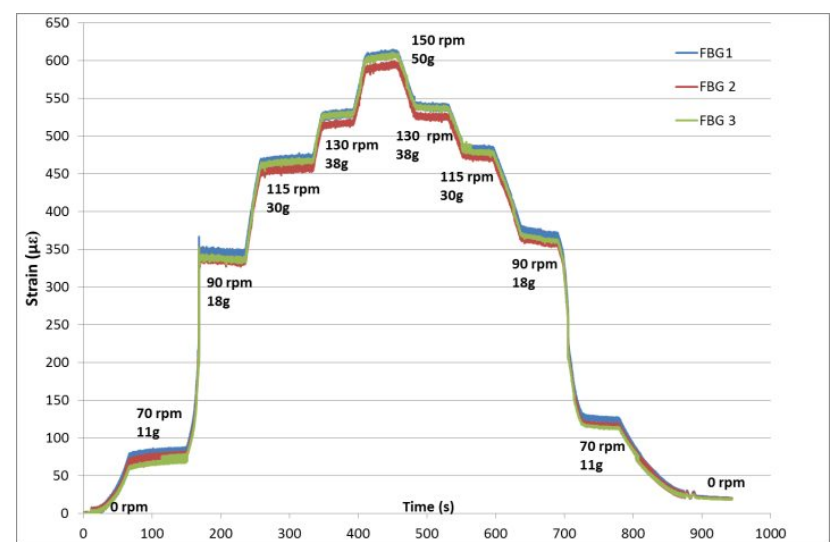

b

Figure 2. a) Changes in the Bragg wavelength of FBG1 measured as the centrifuge was rotated manually; b) The strain measured by the three FBGs as a function of centrifuge rotational speed.

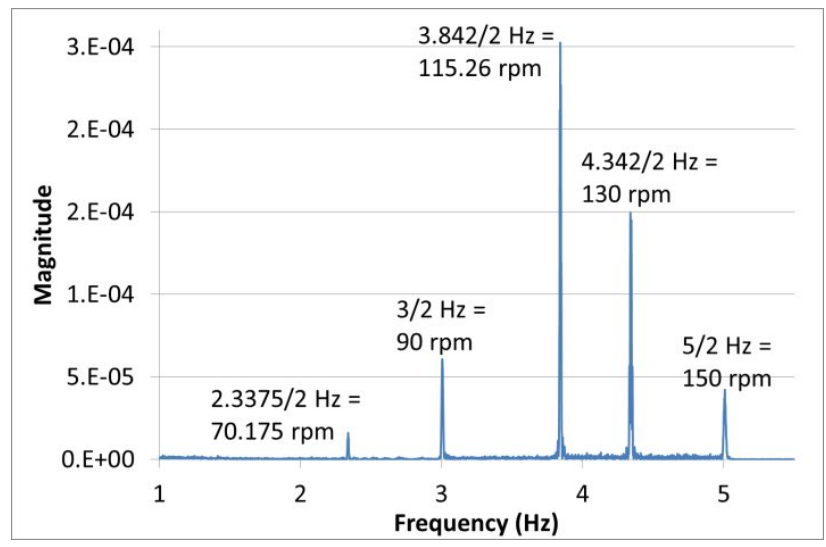

Figure 3. FFT analysis of the tracked FBG peaks as a function of the centrifuge speed. 


\section{CONCLUSION}

The monitoring through a fibre optic rotatory joint of an array of FBG strain sensors attached to a beam that was mounted in a centrifuge was successfully performed. While the low loss of the rotary joint did not compromise the measurement, a periodic intensity modulation imposed by a slight eccentricity in the FORJ was transduced into a false sinusoidal modulation of the Bragg wavelengths, thought to be a result of a limitation of the centroid algorithm used to locate the Bragg peaks.

\section{REFERENCES}

[1] Seaver, M., Trickey, S. T., M. Nichols, J., "Strain Measurements from FBGs Embedded in Rotating Composite Propeller Blades," in Optical Fiber Sensors, OSA Technical Digest (CD), ThD2, (2006).

[2] Chen, Y., Ni, Y. Q., Ye, X. W., Yang, H. X., Zhu, S., "Structural health monitoring of wind turbine blade using fiber Bragg grating sensors and fiber optic rotary joint," Proc. SPIE 8345, Sensors and Smart Structures Technologies for Civil, Mechanical, and Aerospace Systems, 834534, (2012).

[3] Ellis, E. A., Cox, C., Yu, H. S., Ainsworth, A., \& Baker, N. "A new geotechnical centrifuge at the University of Nottingham", UK. In C. W. W. Ng, L. M. Zhang, \& Y. H. Wang (Eds.), Physical Modelling in Geotechnics - 6th ICPMG '06 (pp. 129-133), (2006). 


\section{Interrogation of fibre Bragg gratings through a fibre optic rotary joint on a geotechnical centrifuge}

Correia, Ricardo N.

SPIE

Correia, R. N. (2016) Interrogation of fibre Bragg gratings through a fibre optic rotary joint on a geotechnical centrifuge, 6th European Workshop on Optical Fibre Sensors (EWOFS'2016),

Limerick, Ireland, 30-31 May 2016

http://dx.doi.org/10.1117/12.2236979

Downloaded from Cranfield Library Services E-Repository 